\title{
A REAL-WORLD CASE STUDY OF A VEHICLE ROUTING PROBLEM UNDER UNCERTAIN DEMAND
}

\author{
Anan Mungwattana ${ }^{1}$, Kusuma Soonpracha ${ }^{2}$, Gerrit K. Janssens ${ }^{3}$ \\ ${ }^{1}$ Industrial Engineering Department, Kasetsart University, Bangkok, Thailand \\ ${ }^{2}$ College of Logistics and Supply Chain, Sripatum University, Bangkok, Thailand \\ ${ }^{3}$ Campus Diepenbeek, Research Group Logistics, Universiteit Hasselt University, Hasselt, Belgium
}

Received 16 December 2018; accepted 17 January 2019

\begin{abstract}
The research scope of the real-world logistics industry case study is extended by taking uncertainty in customer demand into account. The particular vehicle routing planning parameters of the logistics provider under study are formulated and are used in two algorithms. The algorithms solve practical problem cases considering a limited number of drivers and a limited company's fleet size but unlimited when considering outsourcing. All trucks are allowed to service multiple trips. The computation is based on real-life data sets. The analysis of the running time and the total transportation cost are compared among three competitive methods. The methods are: the technique based on the company's know-how, a genetic algorithm hybridized with three search operators, and a deterministic annealing hybridized with three search operators. The developed schemes have been proven successful to obtain a near-optimal solution within a reasonable running time. Furthermore, the adaptation of the minimax concept is embedded into the algorithms to find a robust solution for the worst case scenario subject to handling fluctuating situations in demand. In the last phase, two indicators comprising the extra cost and the unmet demand ratios are proposed to help a decision maker to obtain a better view on his decision.
\end{abstract}

Keywords: genetic algorithms, vehicle routing problem, demand uncertainty, logistics, deterministic annealing.

\section{Introduction}

As many companies focus on their core business, warehousing and transport activities are many times outsourced to third-party logistics providers. Road transport is still the most used mode of transport for distribution of goods. Logistics providers want to use their fleet of vehicles in the most efficient way, so route optimisation comes into the picture. In the operations research literature, the Vehicle Routing Problem (VRP) is one of the most studied problems because of both its practical relevance and its computational complexity. The issue concerns the distribution of goods between depots and customers along a set of routes for a fleet of vehicles where an objective function (e.g. total distance, total routing cost) is optimized. Customer demand must be met and vehicle capacities respected. Solving a basic vehicle routing problem involves two elements: the assignment of all customers to a trip and the sequence in which each are visited (Toth and Vigo, 2014).

\footnotetext{
${ }^{1}$ Corresponding author: gerrit.janssens@uhasselt.be
} 
Ideally all input parameters to the optimization problem should be deterministic. However real-life cases face uncertainties in demand, in travel time and in availability of the vehicles. The route planner may be confronted with data disturbances in the routine activities. An optimal solution produced by using deterministic input variables may be infeasible or not good enough in case a situation has changed. In this research, we study a practical case of a heterogeneous fleet vehicle routing problem with real-life constraints under demand uncertainty aiming to fill the gap between recent academic research of vehicle routing problems and transportation industry applications. Two metaheuristic algorithms are developed. A first technique extends the procedure of an evolutionary algorithm using a genetic algorithm from Mungwattana et al. (2016). The literature also indicates that the family of simulated or deterministic annealing algorithms is an attractive methodology to apply for solving VRPs. As a second technique a deterministic annealing algorithms is developed in order to compare its performance against the genetic algorithm and the method based on the company's know-how.

Many of the articles related to the Vehicle Routing Problem are of pure academic nature. The scientific literature publishes from time to time reviews on the evolution of either solution methods for the variants of the VRP or on a classification of various types of VRPs. A recent review can be found in Braekers et al., (2016), in which the interested reader can find references to most of the earlier reviews and classifications. The increase in computing power and the development of efficient algorithms has made that complex variants of the VRP, as they appear in real-life cases, can be solved in a reasonable time. In literature these problems are indicated as 'rich vehicle routing problems' (Hartl et al., 2006, Cruz et al., 2014). Hasle and Kloster (2007) call this type of problems 'industrial or applied vehicle routing'. A need exists in literature to describe solutions for real-life cases with their specific constraints. Some examples of case studies are given in the next paragraph.

Mancini (2015) constructs a search method to assign the best fit refrigerated vehicles starting from different depots to serve customers. In the problem sets, the customer needs are given in units of demand, a solution is feasible as long as the total demand does not exceed the truck capacity. An additional study in the refrigeration logistics is found in Zhang and Chen (2014). The authors extend the classical VRP by allowing the trucks to carry multiple kinds of goods from the same courier. A genetic algorithm is developed to find the optimal solution aiming to minimize the sum of transportation and refrigeration costs. Penalty and cargo damage costs are added to the total cost when some constraints are violated. Sometimes loading constraints are combined with the routing problem. In a study on the fibre board industry, Pace et al. (2015) extended the customer constraints by involving loading methods including stacking limitation, maximum capacity and load balance.

The parameters consisting of customer demand, number of customers, customer/ depot geographical locations, travel times/ travel distances, and transportation costs are the main consideration of most published research. In practice, although other factors such as vehicle availability, resource performance, restricted traffic access, etc. can influence the results. Regarding uncertainties, in literature, most attention

\section{ijtte 102}


has been paid to travel time uncertainty. Stochastic travel times have been studied in e.g. Laporte and Louveaux (1992), Janssens et al. (2009).

This research concentrates on uncertainty in demand. In order to deal with the uncertainty, typically a business sets the rule that customers should submit the demand requirements (orders) in advance. However, in practice, a planner is confronted with the problem that the information is revised frequently by customers. In the company under study, it spends a lot of time and effort to re-compute new solutions to satisfy the new input data set under the various constraints such as time windows, working hour regulations, etc. Besides time spending, the consequence of changes might have an impact on costs such as 1) lost opportunity cost occurs when the company cannot organize the vehicles or manpower resources to serve the new demand additionally to the initial plan, 2) on the contrary, the reserved availability of trucks, rental trucks, or drivers can become idle in case the change is less than in the original planning, 3) the company has to pay a penalty on a monthly basis in case the service performance is lower than the agreement, such indicator is calculated based on actual serviced demands against the total volume of the product delivery request, and so on. Uncertainty in demand has been studied in literature. Demand uncertainty is a serious problem in the VRP as it may lead to unmet demands. Due to the limited vehicle capacity, the main issue is that a vehicle might have to pay an extra visit to the depot for restocking, which requires algorithms for re-optimisation (Haughton, 1998). Exact solutions for the VRP with stochastic demand and customers have been proposed by Gendreau et al. (1995) and Sungur et al. (2008). Metaheuristics like tabu search have been proposed by Gendreau et al. (1996) and particle swarm optimization by Moghaddam et al. (2012).

Earlier, Bräysy et al. (2008) proposed a metaheuristic method adapting the annealing concept to solve the fleet size and mix vehicle routing problem with time windows. In order to speed up the process in the solution improvement phase, the authors implemented multiple local search operators into the deterministic annealing scheme. The neighbourhood search is one of the attractive tools which have been employed in the heterogeneous fleet VRP in order to compose satisfactory solutions. The example of neighbourhood search implementation in the specific vehicle routing problem with time windows and multiple depots was presented in Xu et al. (2012) which uses insert and exchange operators to form a solution. Another adaptation of local neighbourhood search operators has been displayed in Salhi et al. (2014). The authors defined the specific term of a borderline customer, i.e. a customer node located approximately half way between the first and second nearest depots were selected to insert into the prior solution aiming to reduce the cost. An additional real-life case example has been found in Belfiore et al. (2009). The authors defined the mathematical models for the vehicle routing problem with time windows and split deliveries by using a reallife situation of a big retail group in Brazil. The scatter search evolutionary method was introduced targeted to minimize the total distance travelled under the time window constraints. The insert operator was built in the programming to construct the initial solution. The authors took advantage of the scatter search strategy in order to maintain a set of diverse and high quality candidate solutions. The best solution set is 
updated. In this way, the solution was then improved from time to time until reaching the maximum number of iterations.

As described in Mulvey et al. (1995) and Haughton (1998), the robust approach has proven successful to handle uncertainty. The result which is obtained from a robust approach remains close to the optimum, not for one situation but for all cases under consideration. Yin et al. (2009) purposed the sensitivity-based, scenario-based and min-max to determine the robust approaches under the uncertainty of travel demand. Manisri and Mungwattana (2012) proposed three indicators consisting of robust deviation, sum square error, and robust risk criteria to evaluate the solution robustness of a solution to the vehicle routing problem with uncertain travel time due to traffic congestion. The experiment shows that the robustness approach was able to maintain a good performance although it was in the worst case scenario. The Dantzig-Wolfe decomposition technique and a dynamic programming procedure were applied in the algorithm proposed by Lee et al. (2012). Two input parameter sets comprising of travel time and customer demand were considered as being the source of uncertainty. The experiment results suggested that the constructed method of robust approach could generate a satisfactory solution to achieve the target of travel distance minimization.

For this real-life case, customer demand is considered as being uncertain and a worst case approach is considered, i.e. minimax-based, to obtain the robust solution. The last stage is to measure the extra cost and the unmet demand. Both indicators are used to provide additional information for a decision maker in order to evaluate the final outcome. Section 2 introduces the formal problem description.

\section{Problem Description}

The company under study has a total of $N$ customers. The company has one central distribution point. The set of customer nodes is indexed by $j=1,2, \ldots, N$ and node 0 is defined as the depot. Customer $j$ provides the information of its demand requirement of the commodity type $p$ where $p \in P$ to the logistics provider. The planning department consolidates the customer information and determines the routes by assigning an available vehicle $k$ to carry the commodity $p$ to deliver to customer $j$. The company has $|K|$ different types of vehicles $(k \in K)$. A 'vehicle' should be understood as the combination of a truck and a semi-trailer (a semi-trailer truck, hereafter called "truck"). Each type of truck has a known capacity, $m^{p k}$. In case that the company's vehicles cannot fulfil the customers' demands, an outsourcing service by a logistics provider is the second choice. It is assumed that the third party logistics providers have an unlimited number of trucks and can serve all types of products. They can bring a truck into service immediately when the company (the shipper) has a requirement. Besides the truck resource, the number of the company's drivers is another factor which may disrupt the vehicle utilization. According to the policy, only the employees of the company are allowed to drive the company's trucks. In case the required drivers are absent, an outsource provider is contacted to fulfil the customers' demands. The available number of company's drivers is known as $M$. Therefore, the assigned fleet size must be less than or equal to $M$.

Based on the confidential terms of agreement and large demand volumes, these two conditions force all trucks, including the subcontractors' vehicles, to start from and 
return to the central depot after an unloading activity at the customer $j$ site is done. In other words, the transportation activity is a fulltruckload delivery, which means that a truck does not serve more than one customer in a single route. Although unexpected events, such as a road accident or a traffic block, may occur during travelling, this research ignores this factor and makes use of a cycle time $t_{0 j}^{p k}$ representing the expected travel time between the depot and a customer node including the expected service time by the truck $k$ at the customer $j$. In the case study, the customers are located in the same industrial estate and are not far from each other. Therefore, the trucks are allowed to service multiple trips as long as the drivers working hour restriction is not violated. Furthermore, some customers determine limits on the operation time window, and strictly request the truck providing the service within the time windows. Therefore, we consider a period of time, called a time window as being a constraint of this problem. A truck $k$ does not allow entering the customer $j$ site before the earliest opening time, $e_{j}$ or after the latest arrival time, $l_{j}$.

In addition, two factors of the product carried, its weight and dimension limit the semi-trailer's capacity. In practice, each assigned semi-trailer truck can carry one product type to one customer on each trip. The loading capacity of each type of vehicle depends on the product carried which is given by a payload value or an available loadcapacity for each kind of semi-trailer, $q_{j}^{p k}$. The determined value cannot be greater than the truck's capacity. The demand quantity of a customer $j$ is denoted by $D_{j}^{p}$ and is usually beyond the truck's capacity. Therefore, a partial delivery $q_{j}^{p k}$ is accepted by the customers. However, the company must pay penalties to the customer based on business agreement if it cannot schedule the vehicles to serve the total requested demand within the time window. In this study, such a penalty is neglected but all orders are guaranteed to be delivered completely by tightening the constraint. Additionally, due to the fact that the case-study company is confronted with fluctuation in demand, this paper focuses on the real-life case by considering uncertain customer demands. We denote $U d$ to be the set of uncertain demands and define a term of $U d_{j}^{p}=\left(1+\beta_{j}^{s} \alpha_{j}^{s}\right) d_{j}^{0 p}$ to describe the uncertainty model of the customer $j$ where $d_{j}^{0 p}$ is the expected demand of customer $j$. A fluctuating situation in demand $S$ is the combination of different scenario $s$. We assume that the customer demand characteristic is determined by risk aversion $\beta_{j}^{s} \alpha_{j}^{s}$, where $\beta_{j}^{s}$ is an independent random variable and $\alpha_{j}^{s}$ is the perturbation.

Battarra et al. (2009) studied real-world transportation and targeted to obtain the minimum transportation cost for which the trucks can deliver products to the supermarkets in more than one trip. The authors defined the research outline as being a multi-trip vehicle routing problem and set the objective to minimize the number of multiple trips. Although demand uncertainty is considered in our research, each individual scenario is like the classical problem with constant demand. By applying the minimax concept proposed in Kouvelis and Yu (1996), the set of the decision variables is denoted by $X$. The demand data set, $D$ s corresponding to the scenario $s$, is used as being the input variable. The optimal decision $X s^{*}$, related to input $s$ is made towards the objective of minimizing the total number of trips. The set of all feasible decisions against the scenario $s$ is denoted by Fs. 
In this research, the total transportation cost is the combination of two components consisting of a fixed cost and a variable cost. The expenses for which the company pays for the vehicles depreciation, the vehicle insurance, and the vehicle tax are included into the fixed cost. The corporate expenses include the fuel cost which depends on the distance travelled, the driver cost, and the vehicle maintenance cost which varies with mileage used are comprised in the variable cost. The overall fixed and variable costs are combined into a set of vehicle costs $c \in$ $C$ where the vehicle cost $c$ depends on the customer visited, the product served, and the type of vehicle used. Therefore, for this specific problem, the set of decisions $F_{s}$ is defined as the term of $\sum_{j \in N \backslash\{0\}} \sum_{p \in P} \sum_{k \in K} c_{0 j}^{p k} x_{0 j}^{p k}$ , where $c_{0 j}^{p k}$ is the cheapest cost and $x_{0 j}^{p k}$ is the number of trips. The proactive robustness approach is focused to benefit in long run planning by hedging against all scenarios. One of the well known approaches is the absolute robust decision. The absolute robust decisions are of a conservative nature, as they are based on the anticipation that the worst case situation might happen. The solution is made against the best possible outcome under any realizable scenario, and thus bounding the magnitude of missed opportunities, which could be exploited by competitors, in the various scenarios. In this research, the robust decision is modelled as follows:

$$
\begin{aligned}
& \mathrm{z}_{\mathrm{A}}=\max _{\mathrm{s} \in \mathrm{S}} \mathrm{f}\left(\mathrm{X}_{\mathrm{A}}, \mathrm{D}^{\mathrm{s}}\right)= \\
& =\min _{\mathrm{X} \in n_{\mathrm{s} \in \mathrm{S}} \mathrm{F}_{\mathrm{S}}} \max _{\mathrm{s} \in \mathrm{S}}\left(\sum_{j \in N \backslash\{0\}} \sum_{p \in P} \sum_{k \in K} c_{0 j}^{p k} x_{0 j}^{p k}\right)
\end{aligned}
$$

\section{Solution Approach}

A computational method based on the company's know-how (called K-method) serves as being the benchmark problem set in order to compare the performance of two developed techniques. They are: 1) a genetic algorithm hybridized with three search operators (GA-HSO) and, 2) an adapted deterministic annealing hybridized with three search operators (DA-HSO). The structure of both techniques comprises three main procedure steps. First, the initial solution is produced based on the biological evolution mimicry of genetic organisms in the GA-HSO. For the competitive method, DA-HSO, the preliminary solution is obtained from the inspiration of annealing. Second, a solution improvement process is operated. The sub-functions include three operators called 'cross', 'exchange' and 'elimination' operators which are embedded into the solution improvement phase of the algorithms. The first and the second phases are iterated to seek for the best solution for each scenario. In the last step, the minimax technique is applied to find the robust solution.

The above discussion is a general overview of the presented approach. The details of the first technique, called the genetic algorithm hybridized with three search operators (GA-HSO) can be found in Mungwattana et al. (2016). Regarding the new method of the adapted deterministic annealing hybridized with search operators (DA-HSO), this technique is based on the concept of deterministic annealing which makes use of a threshold acceptance criterion. Braekers $e t$ al. (2014) apply the deterministic annealing algorithm to solve a drayage operation in intermodal terminal. The drayage operation is the initial and final part of the intermodal transport chain which is often performed by road and is an example of a vehicle routing problem. Deterministic annealing or threshold acceptance for the vehicle routing problem and variants have been published in Tarantilis et al. (2004); Bräysy 
et al. (2008); Braekers et al. (2011); Caris and Janssens (2010) indicate that outstanding solutions could be obtained by applying a deterministic annealing algorithm. The conceptual idea of threshold accepting value has been initiated by Dueck and Scheuer (1990). This setting is used for determining acceptance criteria to obtain a result. When a neighbor solution is produced, its fitness value is compared with the one of the current solution. A new solution dominates the current one and is accepted to replace the current solution if one of two criteria are satisfied as following; 1) it improves the objective function or 2) its value is smaller than a deterministic threshold accepting setting, is satisfied.

In this research, an adapted deterministic annealing algorithm hybridized with three search operators (DA-HSO) is modified from the scheme as proposed in Caris and Janssens (2010). After the initial solution is generated, the next step is to add number of available vehicles and the number of available driver constraints. An obtained result is called a new solution, sol $_{n}$, or the current solution, sol, and also the best solution, sol $_{b}$, is kept. Three inter-route search operations are applied to randomly search for a new candidate at each iteration. The running loop is terminated when the maximum number of iterations is reached. Similar to the simulated annealing algorithm (SA), a move that reduces the cost function is always accepted. The acceptance rule makes the original simulated annealing procedure differ from the deterministic annealing algorithm (DA). In DA as proposed by Caris and Janssens (2010), the solution is accepted if $\Delta E=C\left(\mathrm{sol}_{n}\right)-C(\mathrm{sol})$ is less than a deterministic threshold value, temp. It is not necessary to make use of probabilies or, by this, random decisions are avoided. The temperature parameter, temp, is set to the maximum temperature, temp $p_{\max }$ when starting the deterministic annealing scheme. It means that the procedure is allowed to accept worse candidate solutions in the beginning of the algorithm.

Three inter-route search operators are implemented for the inside loop of the procedure and are executed repeatedly. A new solution obtained for these sequencing moves, $C\left(\mathrm{sol}_{\mathrm{n}}\right)$, is compared with the current cost value $C(\mathrm{sol})$. If $C\left(\mathrm{sol}_{n}\right)$ is less than $C(\mathrm{sol})$, then the solution is set automatically to the best solution; i.e. $s o l_{b}=s o l_{n}$. In addition, the record of the iteration which found the improvement, iter ${ }_{\text {imp }}$, is updated as equal to the current iteration number, iter. In the case that the new solution is worse than the existing, the current temperature is checked. If the current temperature is less than or equal to zero and the solutions have found no improvement over or equal to the maximum setting; i.e. iter - iter $r_{i m p} \geq n_{\text {no-imp }}$, then the current solution is restarted from the best solution. In each iteration that a new candidate cannot improve the cost function, the temperature is reduced by a small value; temp $=$ temp $-\Delta$ temp. The optimal solution is reached if the cooling is sufficiently slow. After that, resetting the temperature with a random number, rand, where rand $=(0,1)$ and temp $=$ rand. temp $p_{\text {max }}$. The process is repeated until a pre-set number of improved solutions, $n_{i m p}$ is achieved. The overall process of DAHSO is illustrated in Figure 1.

Based on the fact that uncertainties are inherent in the real-world business environment, one of the major issues that the case study company faces is to make the decisions under the situations of changes in demand. The customers may request to add a supplementary quantity to deliver, reduce the required quantity, or cancel 
the order. The solution which has been decided, based on the initial plan under a certain input data set, is optimal for one situation. But such a solution may be not good enough for other realizations in case demand changes. In this study, both methodologies, GA-HSO and DA-HSO, are extended by adding a third phase in order to generate a robust solution. This phase aims to get a reasonable outcome that can hedge against the considered uncertain environments.

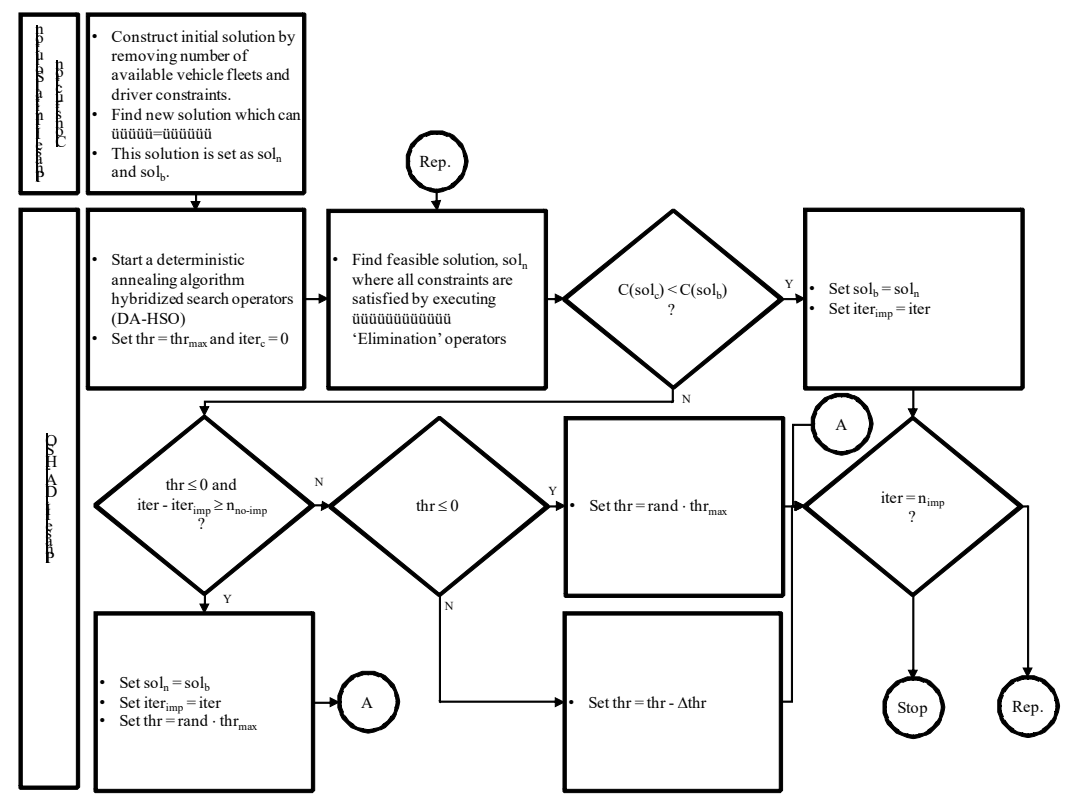

Fig. 1.

The Adapted Deterministic Annealing Algorithm Hybridized with Three Search Operators (DA-HSO)

The target of the first two steps of both the adapted genetic algorithm and the deterministic annealing scheme is to minimize the total cost. A produced solution is stored in a table, called the optimal solution set matrix. After finishing the run for the first scenario, the program reactivates from the beginning step to seek another solution for the next scenario using different input parameters of the customer demand set. The step sequence is iterated until the solutions for all scenarios are obtained. The solutions which are collected in the optimal solution set matrix are recalled to find the robust solution by means of the minimax approach. This approach of Kouvelis and $\mathrm{Yu}$ (1996) has proven to be a great success in many research studies such as Manisri $e t$ al. (2011), Janssens et al. (2015), Soonpracha et al. (2014), Soonpracha et al. (2015), Wang et al. (2017), etc. The absolute robustness measure is one of the minimax decision making models that are used to minimize the objective function, which is the total cost in this problem, among all feasible decisions over all realizable input data scenarios. The diagram of the overall approach is demonstrated in Figure 2. 


\section{Table 1}

Total Cost, Percentage Deviation (\% dev), and Computational Runtime (Avg RT) of GA-HSO and DA-HSO Method Against Company's Knowhow Method (K-method)

\begin{tabular}{|c|c|c|c|c|c|c|c|c|c|c|c|c|}
\hline \multirow[b]{2}{*}{ 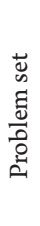 } & \multirow{2}{*}{ 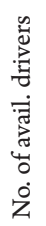 } & \multirow[b]{2}{*}{ 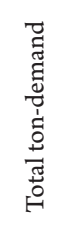 } & \multicolumn{3}{|c|}{ K-method } & \multicolumn{4}{|c|}{ GA-HSO } & \multicolumn{3}{|c|}{ DA-HSO } \\
\hline & & & $\begin{array}{c}\text { Total } \\
\text { cost } \\
\left({ }^{*} 10^{5}\right)\end{array}$ & $\begin{array}{c}\text { Avg RT, } \\
(\mathrm{sec})\end{array}$ & $\begin{array}{l}\text { Total } \\
\text { Cost } \\
\left({ }^{*} 10^{5}\right)\end{array}$ & $\% \operatorname{dev}$ & $\begin{array}{c}\Delta \\
\mathrm{GA}(\%)\end{array}$ & $\begin{array}{c}\text { Avg RT, } \\
(\mathrm{sec})\end{array}$ & $\begin{array}{c}\text { Total } \\
\text { Cost } \\
\left({ }^{*} 10^{5}\right)\end{array}$ & $\% \operatorname{dev}$ & $\begin{array}{c}\Delta \\
\mathrm{DA}(\%)\end{array}$ & $\begin{array}{c}\text { Avg RT, } \\
\text { (sec) }\end{array}$ \\
\hline 1 & 20 & 1,857 & 1.71 & 1,800 & 1.68 & 0.6 & -1.7 & 13 & 1.71 & 0.0 & -0.1 & 15 \\
\hline 2 & 20 & 1,835 & 1.65 & 1,800 & 1.59 & 0.2 & -3.3 & 12 & 1.63 & 0.0 & -1.2 & 13 \\
\hline 3 & 15 & 992 & 0.97 & 1,800 & 0.97 & 0.0 & -0.2 & 10 & 0.97 & 0.0 & -0.2 & 11 \\
\hline 4 & 14 & 1,477 & 1.31 & 1,800 & 1.29 & 0.0 & -1.6 & 12 & 1.29 & 0.0 & -1.6 & 13 \\
\hline 5 & 18 & 1,543 & 1.39 & 1,800 & 1.38 & 0.1 & -0.3 & 11 & 1.39 & 0.0 & -0.1 & 12 \\
\hline 6 & 18 & 1,579 & 1.36 & 1,800 & 1.36 & 0.0 & -0.0 & 10 & 1.36 & 0.0 & -0.0 & 12 \\
\hline 7 & 18 & 1,463 & 1.27 & 1,800 & 1.27 & 0.1 & -0.2 & 10 & 1.27 & 0.0 & -0.1 & 13 \\
\hline 8 & 19 & 1,676 & 1.56 & 1,800 & 1.42 & 0.1 & -8.6 & 11 & 1.45 & 0.0 & -7.0 & 13 \\
\hline 9 & 20 & 1,429 & 1.34 & 1,800 & 1.31 & 0.0 & -2.5 & 11 & 1.31 & 0.0 & -2.5 & 12 \\
\hline 10 & 11 & 745 & 0.70 & 1,800 & 0.69 & 0.0 & -1.2 & 12 & 0.69 & 0.0 & -1.2 & 11 \\
\hline \multicolumn{3}{|c|}{ Avg. } & & 1,800 & & 0.1 & -2.0 & 11 & & 0.0 & -1.4 & 13 \\
\hline
\end{tabular}

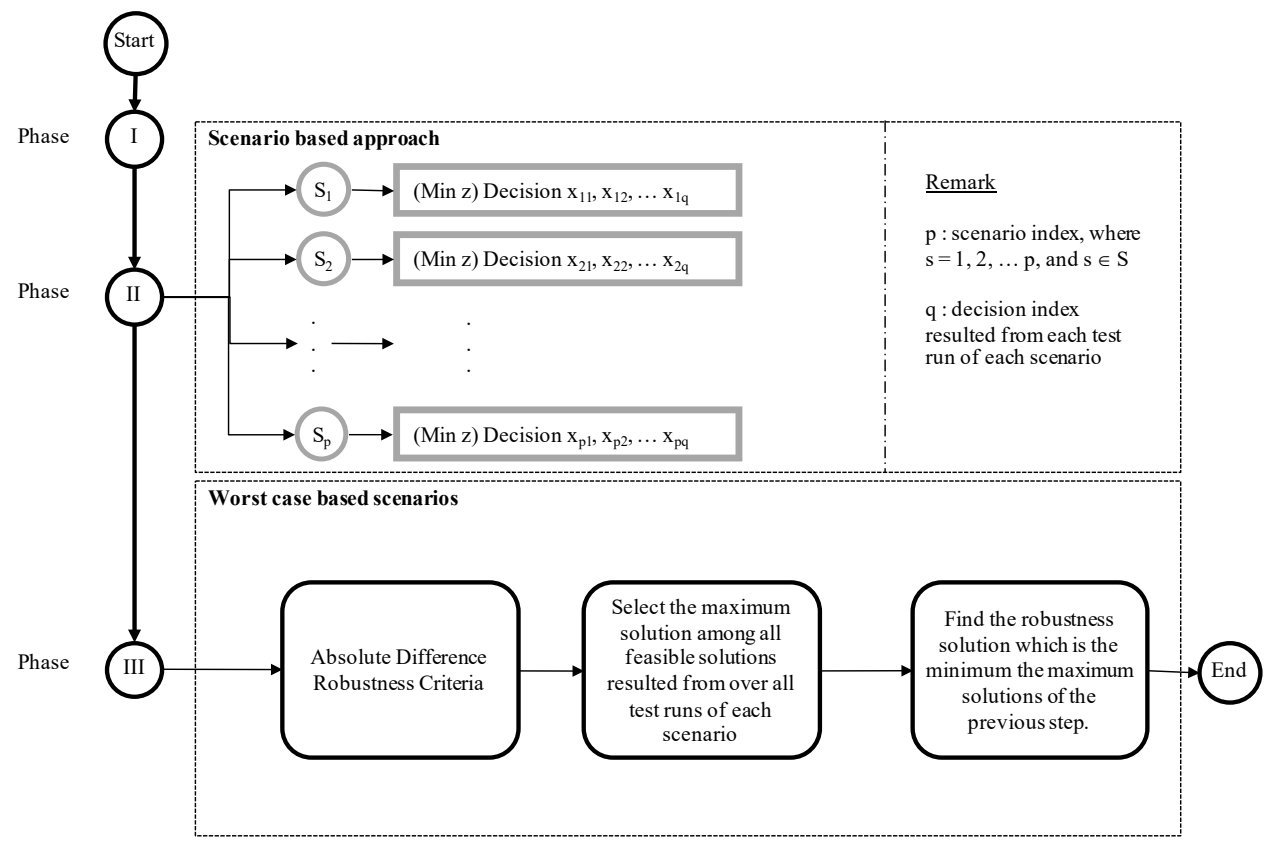

Fig. 2.

Robustness Decision Making 
After the robust result is obtained, its performance is evaluated by two measurement indicators: (1) the extra cost ratio and (2) the unmet demand ratio. These two indicators have been proposed by Sungur et al. (2008). The extra cost ratio (ex) can tell us on the increase of the total cost of the robust solution against the optimal solution obtained by the deterministic approach. The unmet demand ratio $(u)$ suggests the relation between the fixed input data set of the customer demands and the realistic ones which can be faced in the daily routines. These performance indicators are recommended for the practitioners as tools for final decision making. If the total cost of the robust outcome is reasonable but can avoid the potential loss due to unexpected changes in demands, then it guarantees that the solution robustness is good enough as being the answer to cover all realized scenarios. The formulas of both ratios are shown in formulas (2) and (3). The notation of ' $\max U_{d}$ ' refers to the maximum uncertain demand value and $\Sigma d_{i}^{\circ}$ refers to the total demand of all customers which belong to the originally planned data.

$$
\begin{aligned}
& \mathrm{e} x=\frac{z_{\text {Robust }}-z_{\text {Deterministic }}}{z_{\text {Robust }}} \\
& u=\frac{\max U_{d}}{\sum_{i \in C} d_{i}^{\mathbf{0}}}
\end{aligned}
$$

\section{Computational Results}

In this study, two algorithms, GA-HSO and DA-HSO, are proposed to find a sufficiently good solution for a real-world problem case when dealing with several constraints under uncertain demand. The problem set contains thirty customers and a single depot. All customers and depot are located in the same industrial estate. The commodities can be divided into two groups; i.e. a palletized product and a bulk product. The palletized product contains various types of plastic resins which are packed in various sizes of big bags and placed on the pallets. The bulk product contains similar types of the first commodity type, but they are kept in silos. Two main types of trucks with different capacities are assigned to serve both types of commodity.

The own vehicle fleet consists of seventeen semi-trailers and seven bulk trucks. For the bulk product, a specific transfer system is required to feed the product into a truck tank. Such a particular unit is mounted on a bulk truck. Therefore only a bulk truck can serve the bulk product. The semitrailer can be separated into two different parts consisting of a two-axle and a threeaxle chassis. These types of trailers can be allocated to transfer the palletized products to the customers, but cannot carry the bulk product. Furthermore, the loading capacity of the three-axle trailer is larger than the two-axle trailer. In real practice, however, full capacity mostly cannot be utilized due to other restrictions such as container dimension or stacking conditions. Therefore, the company makes agreements with the customers regarding the courier conditions and determines the limitation of payload for each vehicle class.

Sometimes vehicles are not available, for example due to maintenance service. In this study, such situation is considered negligible. However, an additional restriction in this research is related to the number of truck drivers. The maximum number of drivers under a planning-time horizon is equal to twenty, but may be less than the full headcount because of vacation plans. The company drivers operate each type of the firm's trucks. In the case that no 
vehicle truck or driver are available, the contracted outsource service providers form the alternative source to offer service with unlimited resources. Furthermore, transportation activity may face fluctuations in travel time but this study makes use of average cycle times to represent the travel time covering the variation due to unknown disturbances. The logistics provider determines the set of cycle times based on historical records of each round trip to each customer including unloading and loading activities and unexpected events that may occur during transportation. Each vehicle of both the company and the third party logistics provider starts from the depot, travels to only a single customer place, and then returns to the starting point. The nature of all customer demands in this study is larger than the capacity of a single truck. Therefore, the requested delivery quantity can be split in more than one time. However, the planner must manage the route assignments to fulfill the customer demands and satisfy both hard time windows and drivers' working hours constraints.

The GA-HSO and DA-HSO procedures are coded in MATLAB. Ten different data sets are run on an Intel(R) Core(TM) i5-3337U CPU@1.80GHz 8.00GB-RAM. Table 1 illustrates the comparison of output solutions among both developed methods and the company's know-how method (K-method). The table makes use of the expected demand of the initial planning and reports the minimal total transportation cost in which the following conditions are considered: (1) hard time windows, (2) a mix of limited and unlimited number of heterogeneous fleet, (3) a limited number of drivers, (4) split delivery, (5) multiple trips, and (6) capacitated vehicle. According to the planner's approximation, the average computational run time (Avg
$\mathrm{RT}$ ) of the K-method is about thirty minutes per problem set. The Avg RT of GA-HSO and DA-HSO are obtained from the programming report table with 10 runs per problem set. They are equal to 11 and 13 seconds on average. The column on the percentage deviation (\% $\mathrm{dev}$ ) suggests that both proposed algorithms are able to produce a robust solution. The output of each run does not deviate much from each other when the setting parameters of (1) the permutation selection in the evolution operation of the adapted genetic algorithm and (2) the setting temperature in the modified deterministic annealing algorithm, are randomized. Furthermore, the difference in costs (expressed as a percentage) between the $\mathrm{K}$-method and the new methods are denoted by $\triangle \mathrm{GA}(\%)$ and $\triangle \mathrm{DA}(\%)$, when comparing the GA-HSO and the DA-HSO against the K-method, respectively. The application of GA-HSO and DA-HSO for solving the problems helps the business owner to save on the transportation cost about $2 \%$ and $1.4 \%$, respectively.

Although the customer demand is known before the planning process, demand uncertainties regularly appear in real life. This study aims to handle the real situations of the practical cases and assumes that three scenarios can cover all possible situations. The first scenario (Scen-1) uses the original set of customer demand information. The second scenario (Scen-2) is simulated based on the average deviation of the actual demand set of each customer along the planning period, (example shown in Table 2). The convex hull concept as proposed in Sungur et al. (2008) is applied in the third scenario (Scen-3), (the instance displayed in Table 2). Regarding the latter two scenarios, the independent random variable, $\beta_{i}^{S}$, is generated from the interval 
$[-1,1]$ because real life situations suggest that the customers have actual requirements either more than or less than their initial requests. The perturbation parameter, $\alpha_{i}^{S}$ is a value of random number in the range of $(0,1)$.

\section{Table 2}

Example of Uncertain Customer Demand Calculation (Scen-2 and Scen-3)

\begin{tabular}{|c|c|c|c|c|c|c|c|c|c|c|c|c|c|c|c|}
\hline \multirow{2}{*}{\begin{tabular}{l}
$\vec{D}$ \\
$\tilde{0}$ \\
\multirow{0}{*}{} \\
$\tilde{U}$
\end{tabular}} & \multirow[b]{2}{*}{$\sigma^{\circ}$} & \multicolumn{3}{|c|}{ Scen-2 } & \multicolumn{11}{|c|}{ Scen-3 } \\
\hline & & $\infty$ & $\sigma$ & $D^{\sigma}$ & - & $\infty^{2}$ & $\infty^{m}$ & $\sigma^{-1}$ & $\sigma^{N}$ & $\delta^{m}$ & $\widetilde{\sigma}_{-}^{0}$ & $\begin{array}{l}\tilde{\sigma}^{0} \\
\sigma^{N} \\
\sigma^{N}\end{array}$ & $\begin{array}{l}0^{0} \\
\sigma^{m} \\
0^{m}\end{array}$ & $\begin{array}{l}\widetilde{\sigma}^{\circ} \\
\widetilde{\sigma} \\
\stackrel{\omega}{\omega}\end{array}$ & $D^{\sigma}$ \\
\hline 1 & 120 & 1 & 0.2 & 139 & -1 & -1 & 0 & 0 & 0.5 & 0.6 & 0 & -54 & 0 & -54 & 66 \\
\hline 2 & 545 & 0 & 0.8 & 545 & -1 & 1 & 1 & 0.2 & 0.4 & 0.2 & -24 & 48 & 24 & 48 & 593 \\
\hline 3 & 100 & -1 & 0.9 & 15 & 0 & 0 & -1 & 0.8 & 0 & 0.2 & 0 & 0 & -24 & -24 & 76 \\
\hline
\end{tabular}

The obtained solutions of each scenario are stored in the 'optimal solution matrix'. The results in the optimal solution matrix are then used for finding the robust solution. The minimax approach is applied, which assumes that the solution can cover all considered realization scenarios. The robust solution is evaluated based on the absolute difference robustness criterion. The robust solution, which is minimax solution, is obtained as the last step of the procedure. Tables 3 and 4 illustrate the results of one case-studied instance (Problem set no.1 is demonstrated as an example) by applying (1) the adapted genetic algorithm hybridized local search (GA-HSO) and (2) the modified deterministic annealing hybridized local search (DA-HSO). The graphical representation of the total

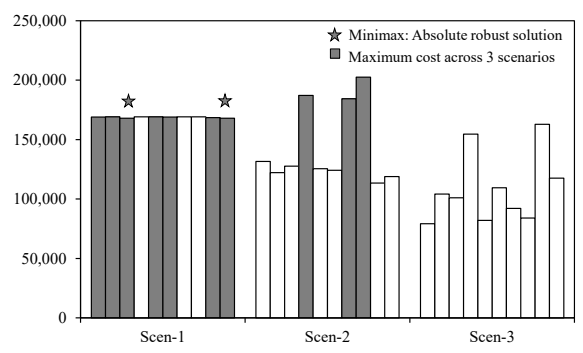

(a) costs of each scenario using GA-HSO and DA-HSO is shown in Figure 3. The robust solutions in this case study are the answer to scenario number 1 , which belongs to the initial plan data set. Most customers of this scenario have an actual demand less than the original orders. Therefore, the algorithms suggest the solutions of the first input of demand sets as being the robust solution which can hedge against uncertainty in all input environments. The algorithms may suggest different answers if the generated scenarios differ from the three example cases. Regarding the computational runtime, the processing times are 13 and 15 seconds for GA-HSO and DA-HSO, respectively, which is a significant improvement when compared with the K-method.

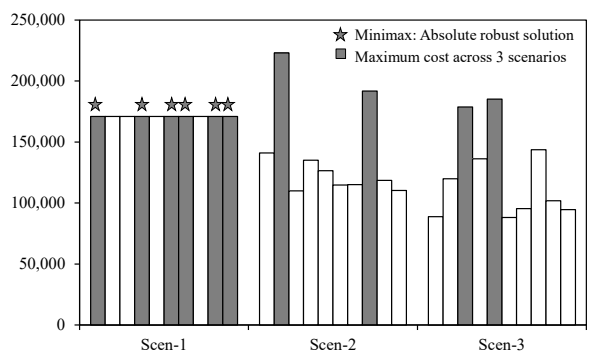

(b)

Fig. 3.

Absolute Robustness Obtained by Applying (a) GA-HSO and (b) DA-HSO 
Table 3

Absolute Robust Solution Obtained by Applying GA-HSO (Problem Set No.1 is for Example.)

\begin{tabular}{|c|c|c|c|c|c|c|c|}
\hline \multirow{2}{*}{$\begin{array}{c}\text { No. of } \\
\text { run }\end{array}$} & \multicolumn{3}{|c|}{ Maximum demand } & \multicolumn{3}{c|}{ Cost } & \multirow{2}{*}{ Absolute robustness } \\
\cline { 2 - 7 } & Scen-1 & Scen-2 & Scen-3 & Scen-1 & Scen-2 & Scen-3 & \\
\hline 1 & 1,857 & 1,324 & 762 & 168,906 & 131,586 & 79,243 & 168,906 \\
\hline 2 & 1,857 & 1,297 & 920 & 169,163 & 122,160 & 104,207 & 169,163 \\
\hline 3 & 1,857 & 1,339 & 891 & 168,011 & 127,653 & 101,045 & $\mathbf{1 6 8 , 0 1 1}$ \\
\hline 4 & 1,857 & 1,936 & 1,651 & 169,163 & 187,096 & 154,523 & 187,096 \\
\hline 5 & 1,857 & 1,359 & 799 & 169,102 & 125,398 & 81,925 & 169,102 \\
\hline 6 & 1,857 & 1,365 & 1,117 & 169,029 & 124,231 & 109,422 & 169,029 \\
\hline 7 & 1,857 & 1,963 & 839 & 169,124 & 184,293 & 92,018 & 184,293 \\
\hline 8 & 1,857 & 2,147 & 773 & 169,124 & 202,422 & 83,996 & 202,422 \\
\hline 9 & 1,857 & 1,161 & 1,752 & 168,445 & 113,401 & 162,797 & 168,445 \\
\hline 10 & 1,857 & 1,224 & 1,104 & 168,011 & 118,928 & 117,500 & $\mathbf{1 6 8 , 0 1 1}$ \\
\hline & & & & & & Minimax & $\mathbf{1 6 8 , 0 1 1}$ \\
\hline
\end{tabular}

Two performance measurements, i.e. the extra cost ratio (ex) and the unmet demand ratio $(u)$ (see Sungur et al., 2008) are considered. They are applied to evaluate the robust solution against the solution obtained from the deterministic approach with constant demand. In this computation, three scenarios represent the whole situation of the uncertainty events which are created based on the risk aversion as presented in Table 2. Under these situations, in order to make use of a robust solution, no extra cost is incurred and the worst case can be handled when the demand is $20 \%$ over the expected plans on average. The performance indicators are reported in Table 5. In this case, the route assignment set under the solution output of the third or the tenth run is suggested to use when GA-HSO is applied for computation. In order to implement this route, the total cost is equal to 168,011 units. DA-HSO produces an output, which is different from the GA-HSO. The solution under the runs with numbers 1 , $4,6,7,9$, or 10 can be chosen as the robust routing plan with a total transportation amount of 170,888 units. By comparing the robust results between GA-HSO with DA$\mathrm{HSO}$, the procedure of DA-HSO is able to produce a higher level of robustness. However, its cost is higher than by using GA-HSO.

\section{Table 4}

Absolute Robust Solution Obtained by Applying DA-HSO (Problem Set No.1 is for Example.)

\begin{tabular}{|c|c|c|c|c|c|c|c|}
\hline \multirow{2}{*}{ No. of run } & \multicolumn{3}{|c|}{ Maximum demand } & \multicolumn{3}{|c|}{ Cost } & \multirow[t]{2}{*}{ Absolute robustness } \\
\hline & Scen-1 & Scen-2 & Scen-3 & Scen-1 & Scen-2 & Scen-3 & \\
\hline 1 & 1,857 & 1,449 & 784 & 170,888 & 140,966 & 88,692 & 170,888 \\
\hline 2 & 1,857 & 2,286 & 1,234 & 170,888 & 222,938 & 119,785 & 222,938 \\
\hline 3 & 1,857 & 1,109 & 1,898 & 170,888 & 109,929 & 178,732 & 178,732 \\
\hline 4 & 1,857 & 1,426 & 1,379 & 170,888 & 135,133 & 136,292 & 170,888 \\
\hline 5 & 1,857 & 1,268 & 1,916 & 170,888 & 126,456 & 185,148 & 185,148 \\
\hline 6 & 1,857 & 1,194 & 746 & 170,888 & 114,772 & 88,105 & 170,888 \\
\hline 7 & 1,857 & 1,134 & 870 & 170,888 & 115,014 & 95,327 & 170,888 \\
\hline 8 & 1,857 & 1,990 & 1,448 & 170,888 & 191,742 & 143,664 & 191,742 \\
\hline 9 & 1,857 & 1,197 & 941 & 170,888 & 118,574 & 101,824 & 170,888 \\
\hline 10 & 1,857 & 1,120 & 887 & 170,888 & 110,291 & 94,591 & 170,888 \\
\hline & & & & & & Minimax & 170,888 \\
\hline
\end{tabular}




\section{Table 5}

Extra Cost Ratio and Unmet Demand Ratio (Problem Set No.1 is for Example.)

\begin{tabular}{|c|c|c|c|c|c|c|}
\hline \multirow{2}{*}{ Algorithm } & \multicolumn{3}{|c|}{ Extra cost ratio, ex } & \multicolumn{3}{c|}{ Unmet demand ratio, $u$} \\
\cline { 2 - 7 } & $\mathrm{Z}_{\text {Robust }}$ & $\mathrm{Z}_{\text {Deterministic }}$ & Ratio $e x$ & $\max U_{d}$ & total $d_{o}$ & Ratio $u$ \\
\hline GA-HSO & 168,011 & 168,011 & 0 & 2,147 & 1,857 & 1.156 \\
\hline DA-HSO & 170,888 & 170,888 & 0 & 2,286 & 1,857 & 1.231 \\
\hline
\end{tabular}

\section{Table 6}

Results of Sensitivity Test - Cost

\begin{tabular}{|c|c|c|c|c|c|c|}
\hline \multirow{2}{*}{ No. of nodes } & \multirow{2}{*}{$\begin{array}{c}\text { Total } \\
\text { demand }\end{array}$} & \multirow{2}{*}{$\begin{array}{c}\text { Total demand when } \\
\text { uncertainty is taken } \\
\text { into account }\end{array}$} & \multicolumn{2}{|c|}{ Solution - Expected demand } & \multicolumn{2}{|c|}{$\begin{array}{c}\text { Absolute Robust solution - } \\
\text { Uncertain demand }\end{array}$} \\
\cline { 5 - 7 } & & 622 & 65,180 & 65,180 & 69,380 & 69,680 \\
\hline 50 & 643 & 3,332 & 241,660 & 241,940 & 245,540 & 255,820 \\
\hline 100 & 2,949 & 2,799 & 270,020 & 270,020 & 283,980 & 283,700 \\
\hline 200 & 2,483 & 3,868 & 401,380 & 401,380 & 415,020 & 420,640 \\
\hline 300 & 3,446 & 4,498 & 511,720 & 511,720 & 545,740 & 537,600 \\
\hline 400 & 4,277 & & & & & DA-HSO \\
\hline
\end{tabular}

\section{Table 7}

Results of Sensitivity Test - Extra Cost, Unmet Demand, and Runtime

\begin{tabular}{|c|c|c|c|c|c|c|}
\hline \multirow{2}{*}{ No. of nodes } & \multirow{2}{*}{ Total demand } & \multirow{2}{*}{$\begin{array}{c}\text { Unmet demand } \\
\text { ratio, } u\end{array}$} & \multicolumn{2}{|c|}{ Extra cost ratio, ex } & \multicolumn{2}{|c|}{ Runtime (seconds) } \\
\cline { 4 - 7 } & & GA-HSO & DA-HSO & GA-HSO & DA-HSO \\
\hline 50 & 643 & 1.15 & 0.064 & 0.069 & 22 & 30 \\
\hline 100 & 2,949 & 1.06 & 0.016 & 0.057 & 42 & 56 \\
\hline 200 & 2,483 & 1.08 & 0.052 & 0.051 & 82 & 108 \\
\hline 300 & 3,446 & 1.09 & 0.034 & 0.048 & 124 & 162 \\
\hline 400 & 4,277 & 1.06 & 0.067 & 0.051 & 172 & 224 \\
\hline \multicolumn{2}{r}{ Average } & 1.09 & 0.047 & 0.055 & & \\
\hline
\end{tabular}

\section{Discussion and Conclusion}

This research relates to a particular realworld implementation by considering uncertainties in demand. Two meta-heuristic optimization methods based on a genetic algorithm and a deterministic annealing algorithm are presented to find robust optimal solutions. Realistic constraints are added to the research to make the problem more complex. The outcomes have been proven to be successful to produce a minimum transportation cost and a robust solution in a short computational time. Tables 6 and 7 illustrate the sensitivity test comparing GA-HSO and DA-HSO methods. Both procedures are limited to apply for solving the problem with a maximum of 400 nodes.

In order to test the performance of the algorithms, all input tables are created using 
random components. In the first scenario, making use of random expected demand sets, both the GA-HSO and DA-HSO schemes suggest route answers which are quite similar. Extra money is added when implementing the robust approach. Another two scenarios are built based on the concept of risk aversion. The GA-HSO procedure suggests the robust solution with an average which is 0.047 times more expensive than the optimal solution with certain demand. The DA-HSO algorithm produces a higher extra cost compared to the GA-HSO method. It is 0.055 times on average more expensive when uncertainties in demand are considered. The robust results are able to handle the unmet demand with a ratio of 1.09 on average. When comparing the length of the computation time, the GA-HSO algorithm always produces the outcomes faster than the DA-HSO method. Both techniques spend a very short computational runtime even when the 400-node case is being tested. Therefore, the strong benefits for the use of both proposed techniques is to advise the sufficiently good route plans under an uncertain demand situation and to shorten time consumed in the routing planning activities.

\section{References}

Agra, A.; Christiansen, M.; Figueiredo, R.; Hvattum, L.M.; Poss, M.; Requejo, C. 2013. The robust vehicle routing problem with time windows, Computers and Operations Research 40(3): 856-866.

Battarra, M.; Monaci, M.; Vigo, D. 2009. An adaptive guidance approach for the heuristic solution of a minimum multiple trip vehicle routing problem, Computers \& Operations Research 36: 3041-3050.

Belfiore, P.; Tsugunobu, H.; Yoshizaki, Y. 2009. Scatter search for a real-life heterogeneous fleet vehicle routing problem with time windows and split deliveries in Brazil, European Journal of Operational Research 199: 750-758.
Braekers, K.; Caris, A.; Janssens, G. K. 2011. A deterministic annealing algorithm for a bi-objective full truckload vehicle routing problem in drayage operations, Procedia Social and Behavioral Sciences 20: 344-353.

Braekers, K.; Caris, A.; Janssens, G. K. 2014. Bi-objective optimization of drayage operations in the service area of intermodal terminals, Transportation Research Part E 65: 50-69.

Braekers, K.; Ramaekers, K.; Van Nieuwenhuyse, I. 2016. The vehicle routing problem: State of the art classification and review, Computers \& Industrial Engineering 99: 300-313.

Bräysy, O.; Dullaert, W.; Hasle, G.; Mest, D. 2008. An effective multirestart deterministic annealing metaheuristic for the fleet size and mix vehicle routing problem with time windows. Transportation Science 42(3): 371-386.

Caceres-Cruz, J.; Arias, P.; Guimarans, D.; Riera, D.; Juan, A.A. 2014. Rich vehicle routing problem: survey, ACM Computing Surveys 47(2): 1-28.

Caris, A.; Janssens, G. K. 2010. A deterministic annealing heuristic for the pre- and end-haulage of intermodal container terminals, International Journal of Computer Aided Engineering and Technology 2(4): 340-355.

Dueck, G.; Scheuer, T. 1990. Threshold accepting: a general purpose optimization algorithm appearing superior to simulated annealing, Journal of Computational Physics 90(1): 161-175.

Gendreau, M.; Laporte, G.; Seguin, R. 1995. An exact algorithm for the vehicle routing problem with stochastic demands and customers, Transportation Science 29(2): 143-155.

Gendreau, M.; Laporte, G.; Seguin, R. 1996. A tabu search heuristic for the vehicle routing problem with stochastic demands and customers, Operations Research 44(3): 469-477. 
Hartl, R.F.; Hasle, G.; Janssens, G.K. 2006. Special issue on rich vehicle routing problems, Central European Journal of Operations Research 14(2): 103-104.

Hasle, G.; Kloster, O. 2007. Industrial vehicle routing. In G. Hasle, K.A. Lie, and E. Quak (eds.). Geometric Modelling, Numerical Simulation, and Optimization, Springer, Berlin, Heidelberg, 397-435.

Haughton, M. A. 1998. The performance of route modification and demand stabilization strategies in stochastic vehicle routing, Transportation Research Part B: Methodological 32(8): 551-566.

Janssens, G.K.; Caris, A.; Ramaekers, K. 2009. Time Petri nets as an evaluation tool for handling travel time uncertainty in vehicle routing solutions, Expert Systems with Applications 36(3): 5987-5991.

Janssens, G. K.; Soonpracha, K.; Manisri, T.; Mungwattana, A. 2015. Robust Vehicle Routing Solutions to Manage Time Windows in the Case of Uncertain Travel Times. In: P. Vasant, ed. Handbook of Research on Artificial Intelligence Techniques and Algorithms. IGI Global, 655-678.

Kouvelis, P.; Yu, G. 1996. Robust discrete optimization and its applications. Dordrecht: Kluwer academic publishers.

Laporte, G.; Louveaux, F.; Mercure, H. 1992. The vehicle routing problem with stochastic travel times, Transportation Science 26(3): 161-170.

Lee, C.; Lee, K.; Park, S.-H. 2012. Robust vehicle routing problem with deadlines and travel time/demand uncertainty, The Journal of the Operational Research Society 63(9): 1294-1306.

Mancini, S. 2015. A real-life multi-depot multi-period vehicle routing problem with a heterogeneous fleet: formulation and adaptive large neighborhood search based matheuristic, Transportation Research Part C Emerging Technologies 70: 100-112.
Manisri, T.; Mungwattana, A. 2012. Comparing the Solutions for Vehicle Routing Problem with Uncertain Travel Times by Robustness Approach, International Journal of Logistics and Transport 63-76.

Manisri, T.; Mungwattana, A.; Janssens, G. K. 2011. Minimax optimisation approach for the robust vehicle routing problem with time windows and uncertain travel times, International Journal of Logistics Systems and Management 10(4): 461-477.

Moghaddam, B. F.; Ruiz, R.; Sadjadi, S. J. 2012. Vehicle routing with uncertain demands: an advanced particle swarm algorithm, Computers \& Industrial Engineering 62(1): 306-317.

Mulvey, J. M.; Vanderbei, R. J.; Zenios, S. A. 1995. Robust optimization of large-scale systems, Operations Research 43(2): 264-281.

Mungwattana, A.; Soonpracha, K.; Manisri, T. 2016. A practical case study of a heterogeneous fleet vehicle routing problem with various constraints. In Proceedings of the 2016 International Conference on Industrial Engineering and Operations Management, Kuala Lumpur, Malaysia, 948-957.

Pace, S.; Turky, A.; Aleti, A.; Moser, I. 2015. Distributing fibre boards: a practical application of the heterogeneous fleet vehicle routing problem with time windows and three-dimensional loading constraints, Procedia Computer Science 51: 2257-2266.

Salhi, S.; Imran, A.; Salhi, S. 2014. The multi-depot vehicle routing problem with heterogeneous vehicle fleet: Formulation and a variable neighborhood search implementation, Computers \& Operations Research 52: 315-325.

Soonpracha, K.; Mungwattana, A.; Manisi, T. 2014. A Three-Phase Algorithm for Solving a Fleet Size and Mix Vehicle Routing Problem with Time Windows Uncertain Demands, Sripatum Review of Science and Technology, 6: 77-89.

\section{jitte 116}


Soonpracha, K.; Mungwattana, A.; Manisri, T. 2015. A re-constructed meta-heuristic algorithm for robust fleet size and mix vehicle routing problem with time windows under uncertain demands. In Proceedings of the $18^{\text {th }}$ Asia-Pacific Symposium on Intelligent and Evolutionary Systems, Springer, 347-361.

Sungur, F.; Ordonez, F.; Dessouky, M. M. 2008. A robust optimization approach for the capacitated vehicle routing problem with demand uncertainty, IIE Transactions 40(5): 509-523.

Tarantilis, C.; Kiranoudis, C.; Vassiliadis, V. 2004. A threshold accepting metaheuristic for the heterogeneous fixed fleet vehicle routing problem, European Journal of Operational Research 152: 148-158.

Toth, P.; Vigo D. 2014. Vehicle Routing: Problems, Methods and Applications ( $2^{\text {nd }}$ ed.), MOS-SIAM Series on Optimization.
Wang, B.; Xia, X.; Meng, H.; Li, T. 2017. Bad-scenario-set Robust Optimization Framework With Two Objectives for Uncertain Scheduling Systems, IEEE/CAA Journal of Automatica Sinica 4(1): 143-153.

Xu, Y.; Wang, L.; Yang, Y. 2012. A New Variable Neighborhood Search Algorithm for the Multi Depot Heterogeneous Vehicle Routing Problem with Time Windows, Electronic Notes in Discrete Mathematics 39: 289-296.

Yin, Y.; Madanat, S.; Lu, X.-Y. 2009. Robust improvement schemes for road networks under demand uncertainty, European Journal of Operational Research 198(2): 470-479.

Zhang, Y.; Chen, X. 2014. An Optimization Model for the Vehicle Routing Problem in Multiproduct Frozen Food Delivery, Journal of Applied Research and Technology 12: 239-250. 